\title{
Strong-coupling Expansions at Finite Temperatures: Application to Quantum Disordered and Quantum Critical Phases
}

\author{
Norbert Elstner \\ Physikalisches Institut, Universität Bonn, Nußallee 12, D-53115 Bonn, Germany \\ Rajiv R. P. Singh \\ Department of Physics, University of California, Davis, California 95616
}

\begin{abstract}
By combining conventional finite-temperature many-body perturbation theory with cluster expansions, we develop a systematic method to carry out high order arbitrary temperature perturbative calculations on the computer. The method is well suited to studying the thermodynamic properties of quantum disordered and quantum critical phases at finite temperatures. As an application, we calculate the magnetic susceptibility, internal energy and specific heat of the bilayer Heisenberg model. It is shown that for a wide range of coupling constants these expansions show excellent convergence at all temperatures. Comparing the direct series ( without extrapolations) for the bulk susceptibility to Quantum Monte Carlo simulations we find an almost perfect agreement between the two methods even at the quantum critical coupling separating the dimerized and antiferromagnetic phases. The convergence fails only at very low temperatures, which are also difficult to reach by Quantum Monte Carlo simulations.
\end{abstract}

Typeset using REVTEX 


\section{INTRODUCTION}

Quantum critical phenomena and quantum disorder in strongly correlated many-body systems have been a subject of much interest lately. They have been invoked to account

for many properties of high temperature superconductors ${ }^{1}$, for non fermi-liquid behavior in heavy fermion materials ${ }^{2}$, and for a variety of other interesting systems ranging from superconductor-insulator transitions to the quantum hall effect ${ }^{3}$. In many problems it is not possible to fine tune the system parameters to be right at the quantum critical point. Nevertheless, the quantum critical point controls their behavior above some very low temperature scale $^{4-6}$. The aim of our study is to develop a method that will allow us to calculate the temperature dependence of thermodynamic quantities with high accuracy for many model Hamiltonians, when the system parameters are in the quantum disordered phase or near a zero temperature quantum critical point.

Quantum spin-systems provide a particularly rich variety of models and real materials where quantum critical phenomena can be studied. There exist many experimental realizations of spin-ladders and alternating chains ${ }^{7}$. Bilayer Heisenberg model is considered relevant to high temperature superconductors ${ }^{8}$. Recently, the material $\mathrm{CaV}_{4} \mathrm{O}_{9}$ has attracted considerable interest $^{9}$. The spins in these materials are arranged in a novel geometrical arrangement, which allows for many possible types of spin-disordered ground states and quantum critical points.

Finite temperature properties of such systems have been studied before by finite-size studies, including exact diagonalization ${ }^{10}$ and Quantum Monte Carlo simulations ${ }^{11,12}$, and by high temperature expansions ${ }^{13}$. The latter method performs poorly at low temperatures for many parameter regimes of interest. Exact diagonalization, in higher than one dimension, is generally limited to fairly small systems and extrapolation to thermodynamic limit is unreliable. The Quantum Monte Carlo method is perhaps the most accurate to date, but it suffers from the sign problem when frustration is present.

Here, we combine many-body perturbation theory with cluster expansions to develop 
high-order strong-coupling expansions for thermodynamic quantities, such as the bulk susceptibility and the specific heat, at arbitrary temperatures. The resulting series are perturbation expansions in the weaker couplings, whose coefficients depend on temperature. For the bilayer Heisenberg model, these series show excellent convergence at all temperatures for a range of parameters ( without using series extrapolation methods such as Pade approximants). Even when the system parameters are tuned to the quantum critical point, the series converges very well down to fairly low temperatures, comparable to the lowest temperatures accessible in the Monte Carlo simulations.

The method should prove useful for frustrated as well as quenched random spin syatems. It can also be applied to electronic models such as t-J and Hubbard or Kondo lattice models. Being a finite temperature perturbation expansion, it is not limited to systems with nondegenerate ground states.

The plan of the paper is as follows: In section II we discuss the basic perturbation theory. In section III we introduce some techniques that are needed for an effective computer implementation of the method. In section IV the series expansions for the bilayer Heisenberg model is presented. In section $\mathrm{V}$ we compare our series with Quantum Monte Carlo simulations. Finally, in section VI we present our conclusions and suggest future directions.

\section{PERTURBATION THEORY}

We are interested in systems which are described by a Hamiltonian

$$
\mathcal{H}=H_{0}+\lambda H_{1}
$$

Here, $H_{0}$ consists of all couplings within an elementary cluster, whereas, $H_{1}$ describes inter-

actions between different such units. The basic idea of cluster expansions is to exploit the fact that in any finite order of perturbation theory only a finite number of these elementary units can be coupled together and thus expansions for a thermodynamic system can be obtained exactly by carrying out perturbation theory just for these few finite-clusters 
or graphs ( In this paper we will refer to them as graphs to distinguish them from the elementary clusters which define the Hamiltonian $H_{0}$ ). A thermodynamic quantity, such as magnetic susceptibility per elementary cluster, $\chi$, for the lattice $\mathcal{L}$ can be written as

$$
\chi(\mathcal{L})=\chi_{0}+\sum_{g} L(g) \times W(g) .
$$

Here $\chi_{0}$ is the susceptibility of the elmentary cluster for $\lambda=0$ and the sum runs over all connected graphs of the lattice. The quantity $L(g)$, called the lattice constant of the graph, is defined as the number of ways per elementary cluster that the graph $g$ can be embedded in the lattice. The quantity $W(g)$ is the weight of the graph $g$ defined by the recursion relation,

$$
W(g)=\chi(g)-N_{g} \chi_{0}-\sum_{g^{\prime}} W\left(g^{\prime}\right),
$$

where, $N_{g}$ is the number of sites in the graph and the sum over $g^{\prime}$ runs over all proper subgraphs of the graph $g$. For a graph $g$ with $B_{g}$ bonds it can be shown ${ }^{14}$ that $W(g)=$ $O\left(\lambda^{B_{g}}\right)$.

So far the formalism is identical to a standard high temperature expansion. The novelty arises in the calculation of $\chi(g)$ for a finite graph. In order to do that, we only need to consider the spins in that graph and the interactions $H_{0}$ and $H_{1}$ between these spins. To calculate expansions at arbitrary temperature, we exploit the following relation

$$
e^{-\beta\left(H_{0}+\lambda H_{1}\right)}=e^{-\beta H_{0}} \sum_{n}(-\lambda)^{n} I_{n}
$$

where $I_{n}$ are n-fold integrals given by,

$$
I_{n}=\int_{0}^{\beta} d t_{1} \int_{0}^{t_{1}} d t_{2} \ldots H_{1}\left(t_{1}\right) H_{1}\left(t_{2}\right) \ldots
$$

Here, the operators, $H_{1}(t)$ have the standard time dependence of the interaction representation

$$
H_{1}(t)=e^{t H_{0}} H_{1} e^{-t H_{0}}
$$

Thus, the partition function can be reduced to the expression, 


$$
Z=Z_{0}+\sum_{n=1}(-\lambda)^{n} Z_{n}
$$

where $Z_{n}$ are given by

$$
Z_{n}=\int_{0}^{\beta} d t_{1} \int_{0}^{t_{1}} d t_{2} \ldots \operatorname{Tr}\left[e^{-\beta H_{0}} H_{1}\left(t_{1}\right) H_{1}\left(t_{2}\right) \ldots\right] .
$$

It is evident that in order to evaluate these expressions, we need to work in a basis in which $H_{0}$ is diagonal. This basis is simply a direct product of the eigen-basis for elementary clusters. Thus calculating thermodynamic quantities is straightforward if the matrix elements of $H_{0}$

and $H_{1}$ are known in this basis. In the next section we discuss an efficient method for calculating these traces and integrals.

\section{EVALUATING THE MULTIPLE INTEGRALS}

The basic energy scale in this problem is set by the level spacing $\Delta_{0}$ in the spectrum of the unperturbed part $H_{0}$. Measuring temperature in units of this quantity one finds that calculating $Z_{n}$ requires repeated integrations over functions of type:

$$
I\left(k, l ; x_{\nu}\right)=x_{\nu}^{k} e^{l x_{\nu}}
$$

Where $k$ and $l$ are integers and $k \geq 0$. It is then easy to see that the $I(k, l ; x)$ form a closed set, because

$$
\begin{aligned}
l \neq 0: & \\
\int_{0}^{x_{\nu-1}} d x_{\nu} x_{\nu}^{k} e^{l x_{\nu}} & =k !\left(\frac{-1}{l}\right)^{k+1} \\
& +\sum_{i=0}^{k}(-1)^{i} \frac{1}{l^{i+1}} \frac{k !}{(k-i) !} x_{\nu-1}^{k-i} e^{l x_{\nu-1}} \\
l=0: & \\
\int_{0}^{x_{\nu-1}} d x_{\nu} x_{\nu}^{k} & =\frac{1}{k+1} x_{\nu-1}^{k+1}
\end{aligned}
$$

These equations allow for an iterative evaluation of the multiple integrals entering Eqn.(8). One finds that the coefficients $Z_{n}$ are finite polynomials in the two variables $x=\Delta_{0} / k_{\mathrm{B}} T$ and $y=\exp \left(-\Delta_{0} / k_{\mathrm{B}} T\right)$. 
In the following we will apply this series expansion method to systems where the basic cluster consists of a pair of $s=1 / 2$ spins coupled by a Heisenberg exchange $J_{\perp}$. Thus, $\Delta_{0}$ is the singlet-triplet spacing in the spectrum of $H_{0}$. In this case it turns out to be more convenient to use the variables $\left(x, Z_{0}\right)$ instead of the variables $(x, y)$, where $Z_{0}=1+3 y$, is the zeroth order partition function.

\section{MODEL AND TABLES}

Here, we apply the method to the spin-half bilayer Heisenberg model, defined by the Hamiltonian

$$
\mathcal{H}=J_{\perp} \sum_{i} \mathbf{S}_{A, i} \cdot \mathbf{S}_{B, i}+J_{\|} \sum_{\langle i, j\rangle} \mathbf{S}_{A, i} \cdot \mathbf{S}_{A, j}+\mathbf{S}_{B, i} \cdot \mathbf{S}_{B, j}
$$

Here, the index $i$ enumerates sites on a two dimensional square lattice and $\langle i, j\rangle$ are pairs of nearest neighbour sites on this lattice. The expansion parameter is given by ratio of the inter dimer to the intra dimer coupling:

$$
\lambda=J_{\|} / J_{\perp}
$$

The series for the susceptibility $\chi$ and the logarithm of the partition function $\ln Z$ per dimer are presented in Tables 1 and 2. The series can also be obtained on the WWW. The acess adress is given at the end of this article.

\section{RESULTS AND COMPARISONS}

In this section we show the convergence of the expansions by comparing partial sums of different order and by comparing with the Quantum Monte Carlo data. It is known from a number of studies at $T=0$ that this model has a quantum critical point at $\lambda \approx 0.4^{11,15}$. We show here results in the quantum disordered phase ( at $\lambda=0.3$ ) and near the quantum critical point at $\lambda=0.4$. 
In figures (1) and (2) the susceptibility and specific heat are plotted as a function of temperature for $\lambda=0.3$. One can see that there is excellent convergence at all temperatures.

The quantum critical regime was investigated by Chubukov, Sachdev and $\mathrm{Ye}^{5}$ by a large$\mathrm{N}$ expansion of the quantum nonlinear $\sigma$-model. They obtained very detailed results for the low temperature behaviour. In particular the following predictions for the susceptibility and the specific heat per unit cell were derived:

$$
\begin{aligned}
\chi & =\frac{k_{\mathrm{B}} T}{(\hbar c)^{2}} \frac{\sqrt{5}}{\pi} \ln \left(\frac{\sqrt{5}+1}{2}\right)\left[1-\frac{0.6189}{N}+O\left(N^{-2}\right)\right] \\
C & =\frac{3 \zeta(3)}{\pi} k_{\mathrm{B}}\left(\frac{k_{\mathrm{B}} T}{\hbar c}\right)^{2} N\left[\frac{4}{5}-\frac{0.3344}{N}+O\left(N^{-2}\right)\right]
\end{aligned}
$$

Where $c$ is the spin wave velocity. From various zero temperature calculations its value is known to be ${ }^{15}$ :

$$
C=1.90 J_{\|}
$$

Evaluating these equations for $N=3$ gives for the susceptibility and specific heat per site:

$$
\begin{aligned}
\chi & =\frac{1}{2 \lambda^{2}} 0.272 k_{\mathrm{B}} T \\
C & =\frac{1}{2 \lambda^{2}} 2.371 k_{\mathrm{B}}\left(k_{\mathrm{B}} T\right)^{2}
\end{aligned}
$$

Here temperature is in units of the intra dimer coupling $J_{\perp}$.

A very sensitive measure for quantum criticality is the Wilson ratio defined by

$$
W=\frac{k_{\mathrm{B}}^{2} T \chi(T)}{C(T)} .
$$

The numerical value of this dimensionless quantity follows immediately from Eqns.(17) and (18):

$$
W=0.115 \quad \text {. }
$$

In figures $3,4,5$, and 6 , the susceptibility, internal energy, specific heat, and Wilson ratio for the model are shown and compared with Monte Carlo data and with the asymptotic quantum critical predictions. We see that the series converges extremely well down to fairly 
low temperatures. However, the convergence appears to break down just as the asymptotic quantum critical behavior sets in. The finite series become oscillatory at these very low temperatures. The activated behaviour at extremly low temperatures as seen in figures 3 , 5 and 6 is also an artifact of finite series. In principle various extrapolation techniques, e.g. Pade approximants, can be used to enhance the convergence in this region. Here, we restrict the analysis to the temperature range where the series converge.

One interesting result to emerge from this study is that asymptotic quantum critical scaling occurs in the model only at very low temperatures, much lower than anticipated before. The uniform susceptibility between $T=0.1 J_{\perp}$ and $T=0.4 J_{\perp}$ appears nearly linear but is not strictly so. There is a shoulder around $T=0.2 J_{\perp}$, which is present in both the series results and the Monte Carlo data.

On the other hand, many amplitude ratios follow the universal quantum critical predictions even above $T=0.1 J_{\perp}{ }^{12}$. Thus, the extent of the quantum critical regime in $2 \mathrm{D}$ systems appears to strongly depend on the quantity studied. Clearly, the role of lattice corrections needs to be better understood ${ }^{16}$.

\section{CONCLUSIONS AND FUTURE DIRECTIONS}

In this paper we presented a practical method to calculate high order strong coupling expansions for quantum statistical models at arbitrary temperatures. The method was applied to the bilayer Heisenberg model, where it shows excellent convergence even near the quantum critical point. In a companion paper, we will discuss application of these methods to alternating spin-chains and spin-ladders. The method is also applicable to frustrated and quenched random spin models as well as to Hubbard or Kondo models around the strong coupling limit. We are preparing to pursue these calculations in the future.

Series for the susceptibility $\chi$, internal energy $E$ and specific heat $C$ may be obtained on the WWW. In addition a Fortran program to read the data files and sum the series is also available. 
The WWW access is via http://brahms.physik.uni-bonn.de/ norbert/series/series.html Acknowledgements:

We are greatful to A. Sandvik for providing the QMC data. One of us (NE) acknowledges the hospitality of the University of California at Davis where part of this work was done. This work is supported in part by the US National Science Foundation under Grant No. DMR-96-16574 (RRPS). 


\section{REFERENCES}

${ }^{1}$ A. Sokol and D. Pines, Phys. Rev. Lett. 71, 2813 (1993); A. V. Chubukov, S. Sachdev and A. Sokol, Phys. Rev. B49, 9052 (1994).

${ }^{2}$ M. C. Aronson et al., Phys. Rev. Lett. 75, 725 (1995).

${ }^{3}$ S. L. Sondhi, S. M. Girvin, J. P. Carini and D. Sahar, Rev. Mod. Phys. 69, 315 (1997).

${ }^{4}$ S. Chakravarty, B. I. Halperin and D. R. Nelson, Phys. Rev. B 39, 2344 (1989).

${ }^{5}$ A. V. Chubukov, S. Sachdev and J. W. Ye, Phys. Rev. B 49, 11919 (1994); S. Sachdev and J. W. Ye, Phys. Rev. Lett. 69, 2411 (1992).

${ }^{6}$ N. Elstner, Int. J. Mod. Phys. B 11, 1753 (1997).

${ }^{7}$ E. Dagotto and T. M. Rice, Science 271, 618 (1996).

${ }^{8}$ A. J. Millis and H. Monien, Phys. Rev. B50, 16606 (1994); H. Monien and T. M. Rice, Physica C, 1705 (1994).

${ }^{9}$ K. Udea et al. Phys. Rev. Lett. 76, 1932 (1996); M. Troyer et al. Phys. Rev. Lett. 76, 3822 (1996). O. A. Starykh et al., Phys. Rev. Lett. 77, 2558 (1996). M. P. Gelfand et al., Phys. Rev. Lett. 77, 2794 (1996). W. E. Pickett, Phys. Rev. Lett. 79, 1746 (1997).

${ }^{10}$ J. Jaklic and P. Prelovsek, Phys. Rev. Lett. 77, 892 (1996).

${ }^{11}$ A. W. Sandvik and D. J. Scalapino, Phys. Rev. Lett. 72, 2777 (1994)

${ }^{12}$ A. W. Sandvik, A. V. Chubukov and S. Sachdev, Phys. Rev. B51, 16483 (1995).

${ }^{13}$ J. Oitmaa et al., 54, 1009 (1996); W. H. Zheng et al., Phys. Rev. B 55, 11377 (1997).

14 M. P. Gelfand, R. R. P. Singh and D. A. Huse, J. Stat. Phys. 59, 1093 (1990).

${ }^{15}$ K. Hida, J. Phys. Soc. Jpn 61, 1013 (1992); M. P. Gelfand, Phys. Rev. B 53, 11309 (1996); W. H. Zheng Phys. Rev. B55, 12267 (1997). 
${ }^{16}$ N. Elstner, R. L. Glenister, R. R. P. Singh and A. Sokol, Phys. Rev. B51, 8984 (1995). 


\section{FIGURES}

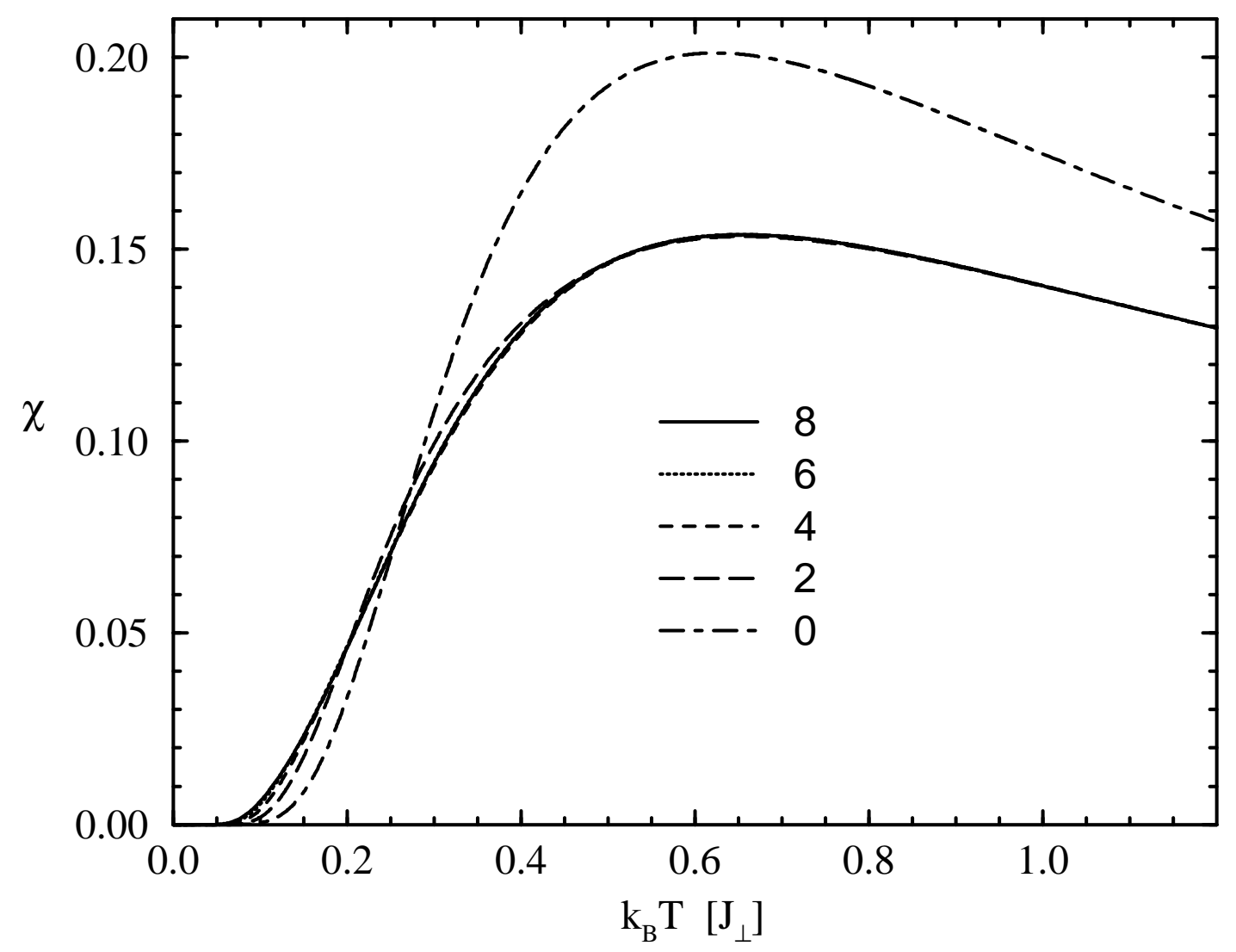

FIG. 1. Susceptibility $\chi$ per spin vs. temperature $k_{\mathrm{B}} T / J_{\perp}$ for $J_{\|} / J_{\perp}=0.3$, i.e. in the quantum disordered regime. The lines are series of order 2, 4, 6 and 8 compared to the susceptibility of an isolated dimer (0). 


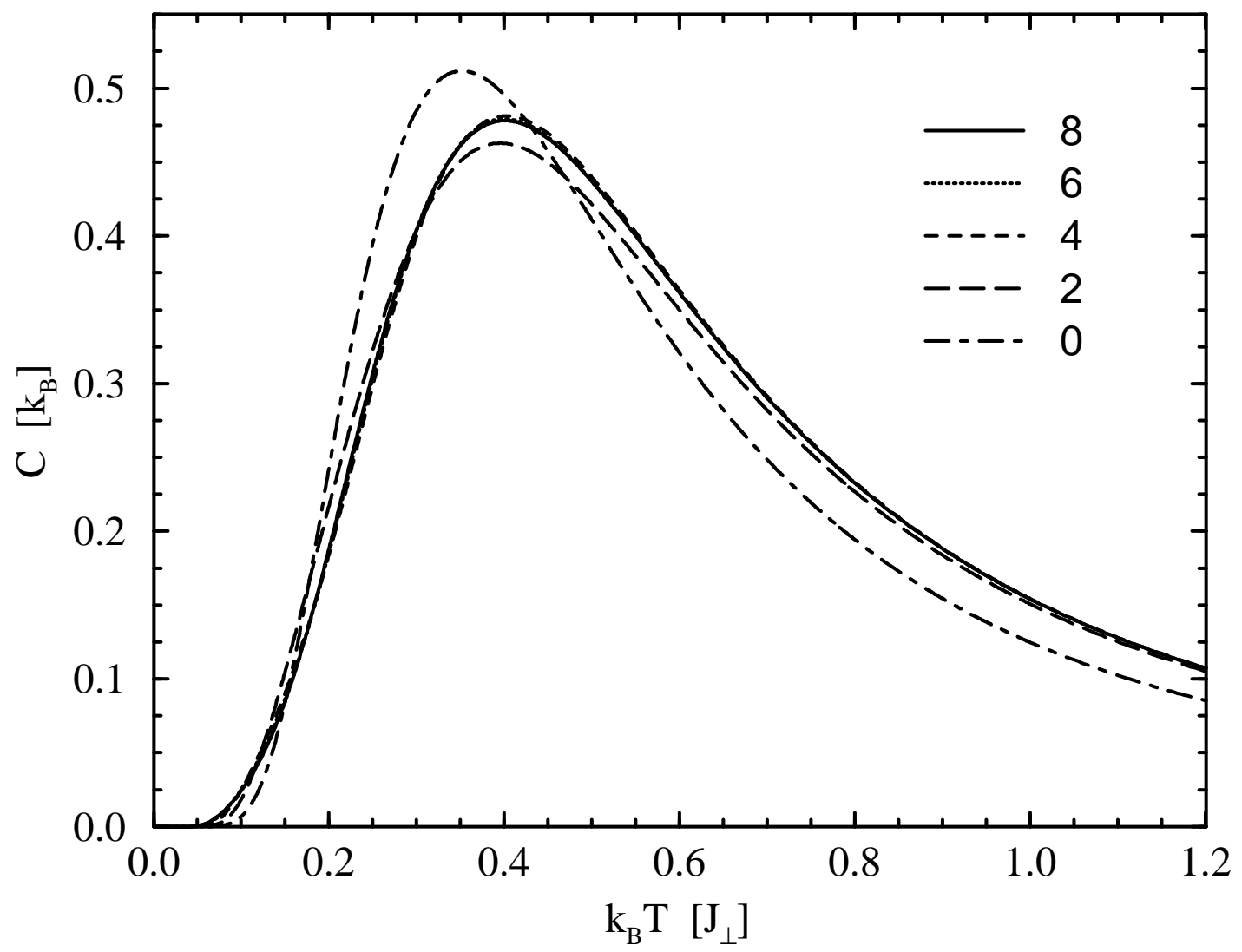

FIG. 2. Specific heat $C$ per spin vs. temperature $k_{\mathrm{B}} T / J_{\perp}$ for $J_{\|} / J_{\perp}=0.3$, i.e. in the quantum disordered regime. The lines are series of order 2, 4, 6 and 8 compared to the specific heat of an isolated dimer $(0)$. 


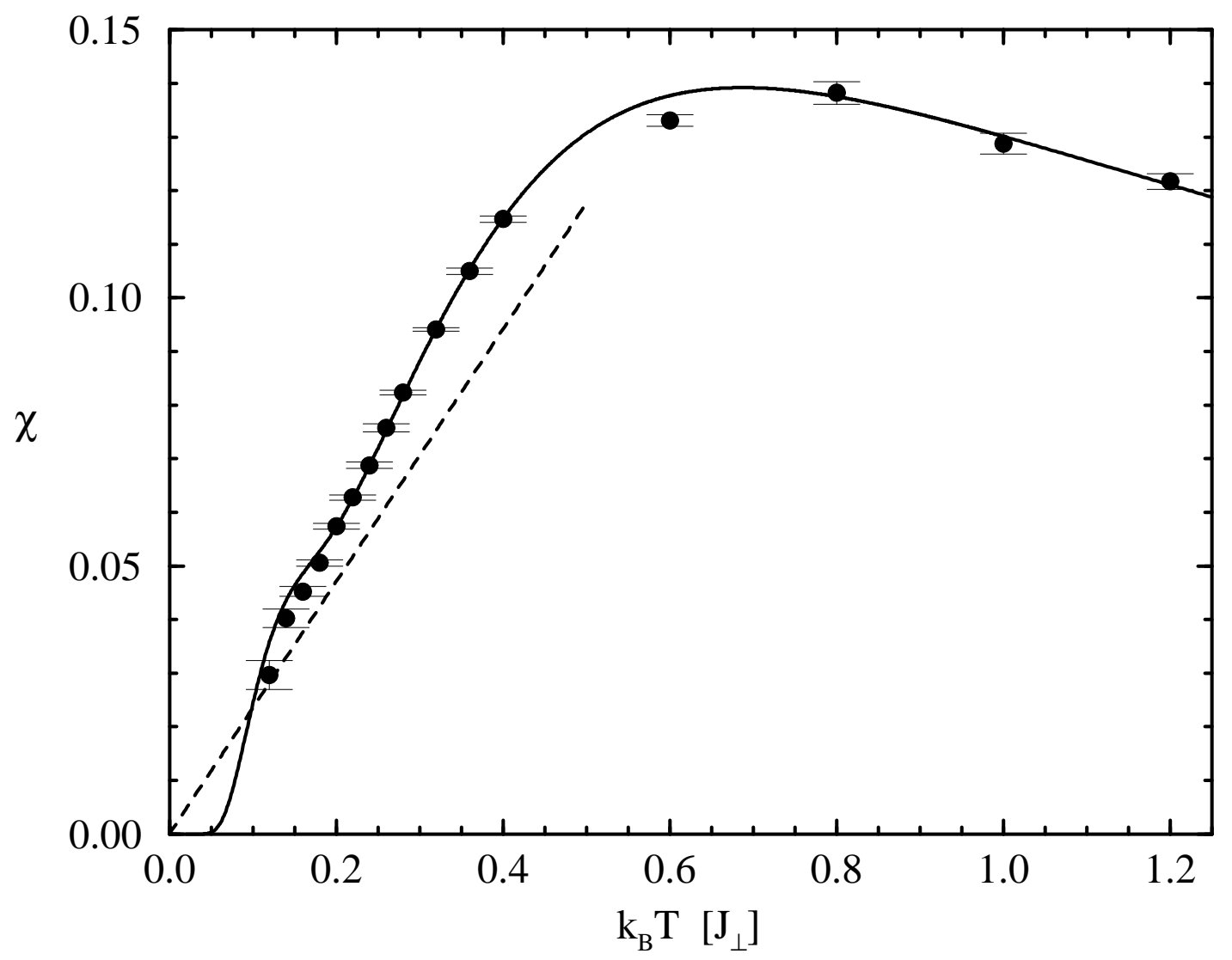

FIG. 3. Susceptibility $\chi$ per spin vs. temperature $k_{\mathrm{B}} T / J_{\perp}$ for $J_{\|} / J_{\perp}=0.4$, i.e. close to the quantum critical point. The black circles show the QMC data of Sandvik and Scalapino. The solid lines is the series of order 8. The dashed line is the quantum critical (QC) prediction of Eqn. (17) 


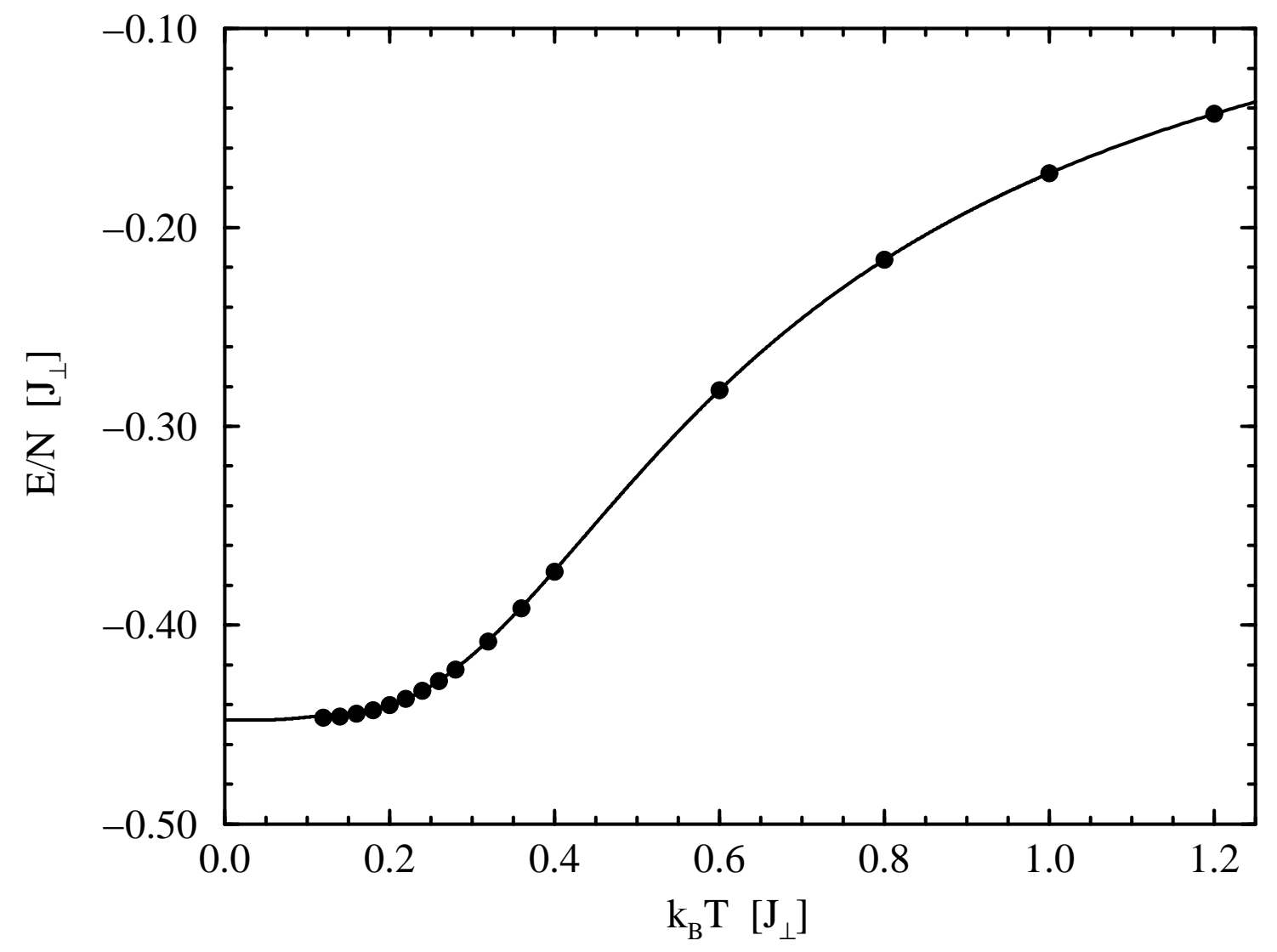

FIG. 4. Internal Energy per spin $E / N J_{\perp}$ vs. temperature $k_{\mathrm{B}} T / J_{\perp}$ for $J_{\|} / J_{\perp}=0.4$. The line is the series up to order 8. The black dots show the QMC data of Sandvik and Scalapino. 


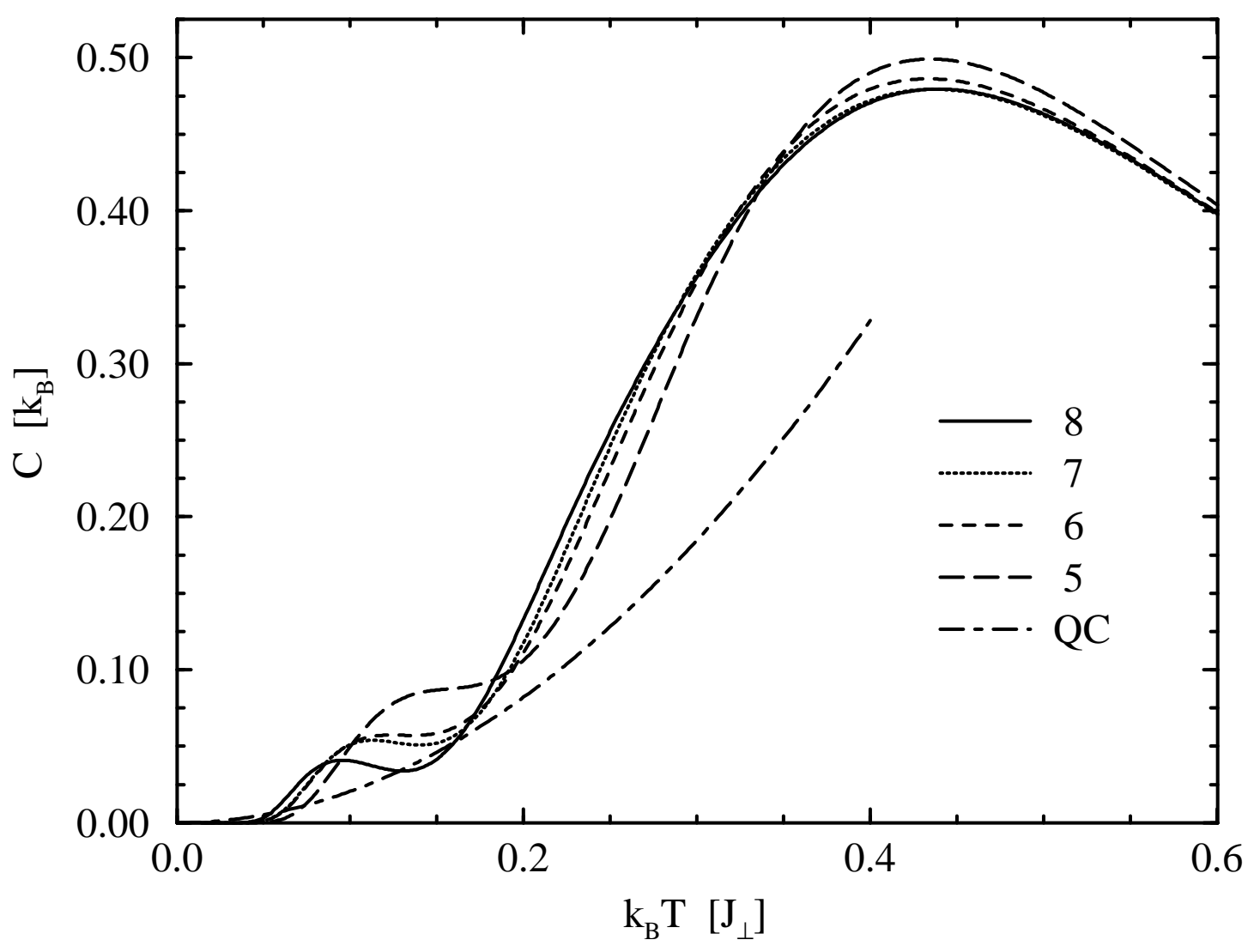

FIG. 5. Specific heat per spin $C$ vs. temperature $k_{\mathrm{B}} T / J_{\perp}$ for $J_{\|} / J_{\perp}=0.4$. The lines are series of order 5, 6, 7 and 8 and the quantum critical (QC) prediction of Eqn. (18) . 


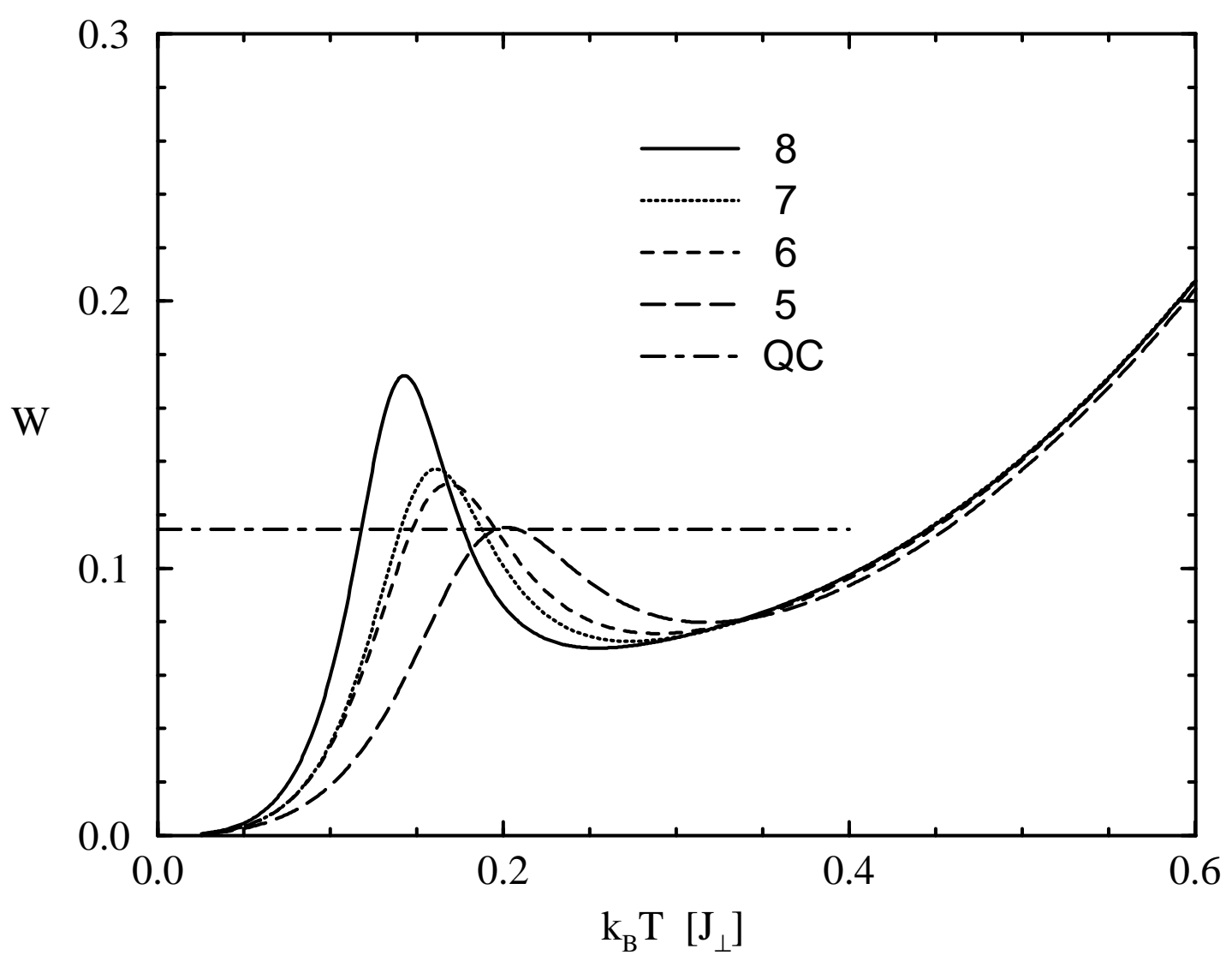

FIG. 6. Wilson ratio $W$ vs. temperature $k_{\mathrm{B}} T / J_{\perp}$ for $J_{\|} / J_{\perp}=0.4$. $W$ obtained from series for $\chi$ and $C$ of order 5, 6, 7 and 8 and compared to the quantum critical (QC) prediction of Eqn. (20) 
Table 1.: Series for the bulk susceptibility $\chi$ per dimer

$$
\chi_{u}(\lambda ; \beta)=\frac{1}{12} \sum_{n=0}(-\lambda)^{n} \frac{1}{12^{n} n !} \chi_{n}(\beta) \quad \text { with } \quad \chi_{n}(\beta)=\sum_{k=0}^{n} \sum_{l=0}^{n+1} c_{k l} \beta^{k} Z_{0}^{-l} .
$$

Here $Z_{0}=1+3 \exp (-\beta)$ is the partition function of an isolated dimer.

$\chi_{0}:$

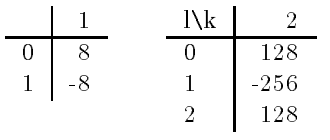

$\chi_{2}$ :

\begin{tabular}{l|r|r}
$\mathrm{l} \backslash \mathrm{k}$ & 2 & 3 \\
\hline 0 & 384 & 2688 \\
1 & -1152 & -8064 \\
2 & -2304 & 9216 \\
3 & 3072 & -3840
\end{tabular}

\begin{tabular}{l|r|r|r}
$\mathrm{l} \mathrm{k}$ & 2 & 3 & 4 \\
\hline 0 & -6912 & 18432 & 73728 \\
1 & 20736 & -87552 & -308736 \\
2 & 41472 & -138240 & 571392 \\
3 & -55296 & 465408 & -511488 \\
4 & 0 & -258048 & 175104
\end{tabular}

$\chi_{4}$ :

\begin{tabular}{l|r|r|r|r}
$l \backslash \mathrm{k}$ & 2 & 3 & 4 & 5 \\
\hline 0 & 787968 & 428544 & 912384 & 2370048 \\
1 & -4280832 & -2032128 & -4396032 & -11976192 \\
2 & 2497536 & 14515200 & -6027264 & 28821504 \\
3 & 13381632 & -42771456 & 41969664 & -40270848 \\
4 & -24182784 & 51093504 & -54577152 & 30504960 \\
5 & 11796480 & -21233664 & 22118400 & -9449472
\end{tabular}

$\chi_{5}$ :

\begin{tabular}{l|r|r|r|r|r}
$1 \backslash \mathrm{k}$ & 2 & 3 & 4 & 5 & 6 \\
\hline 0 & -57646080 & 14837760 & 29767680 & 44605440 & 87932928 \\
1 & 346982400 & -165703680 & -195010560 & -221921280 & -476614656 \\
2 & -273576960 & -428359680 & 1591050240 & -441077760 & 1352337408 \\
3 & -1213470720 & 3835146240 & -6198865920 & 3826851840 & -2642288640 \\
4 & 1976279040 & -7331604480 & 11384985600 & -7836364800 & 3361351680 \\
5 & -778567680 & 5491261440 & -9726197760 & 6798458880 & -2349711360 \\
6 & 0 & -1415577600 & 3114270720 & -2170552320 & 666992640
\end{tabular}

$\chi_{6}:$

\begin{tabular}{l|r|r|r|r|r|r}
$1 \backslash \mathrm{k}$ & 2 & 3 & 4 & 5 & 6 & 7 \\
\hline 0 & 12918528000 & 7489013760 & 4882083840 & 2302248960 & 2285660160 & 3858315264 \\
1 & -83086663680 & -37391155200 & -29798461440 & -16123207680 & -13725573120 & -23258603520 \\
2 & 112199178240 & 219853854720 & -64553656320 & 138725775360 & -22857431040 & 71384205312 \\
3 & 145302958080 & -829322219520 & 682678886400 & -639576207360 & 278069760000 & -155211411456 \\
4 & -506555596800 & 1734012334080 & -1915608268800 & 1637361561600 & -784237731840 & 258483916800 \\
5 & 696756142080 & -2181593088000 & 2706940477440 & -2308508467200 & 1093588992000 & -294514016256 \\
6 & -591569879040 & 1553242521600 & -1938315018240 & 1653277409280 & -767088230400 & 190193614848 \\
7 & 214035333120 & -466291261440 & 553773957120 & -467459112960 & 213964554240 & -50936020992
\end{tabular}




\begin{tabular}{l|r|r|r|r|r|r|r}
$1 \backslash \mathrm{k}$ & 2 & 3 & 4 & 5 & 7 & 8 \\
\hline 0 & -2213251568640 & -495870197760 & 327807406080 & 350493696000 & 169998151680 & 133894397952 & 188246827008 \\
1 & 14563573355520 & 1073517096960 & -2334586060800 & -2449236787200 & -1446944808960 & -920540823552 & -1267894665216 \\
2 & -21593862420480 & -27276743208960 & 34889907548160 & -7746371297280 & 11662847078400 & -207016943616 & 3808230875136 \\
3 & -23096475924480 & 140663776296960 & -173864730009600 & 95048378449920 & -63978511564800 & 16089805799424 & -7204267671552 \\
4 & 100188984913920 & -337715756421120 & 467620165877760 & -348518405652480 & 214780183511040 & -65438937317376 & 12744739897344 \\
5 & -132076661391360 & 476530149150720 & -768271630049280 & 683237322915840 & -426119730462720 & 136632715591680 & -22588348317696 \\
6 & 90411348787200 & -401291848581120 & 752002630041600 & -755460220354560 & 483148426199040 & -160560949248000 & 27719857815552 \\
7 & -26183655751680 & 182473048719360 & -395769662668800 & 440930622504960 & -288393102950400 & 99145204236288 & -18112389120000 \\
8 & 0 & -33960272855040 & 85400097914880 & -105392583475200 & 70176834846720 & -24874175692800 & 4711824359424
\end{tabular}

$\chi_{8}:$

\begin{tabular}{|c|c|c|c|c|c|c|c|c|}
\hline $\mathrm{k}$ & 2 & 3 & 4 & 5 & 6 & 7 & 8 & \\
\hline$\overline{0}$ & 87200 & 920 & 920 & 60667304140800 & 24452127866880 & 9493653012480 & 8024287444992 & 9676674736128 \\
\hline 1 & 3899520 & 383360 & 1060697318768640 & 71476480 & 54240 & 170432 & 909952 & 641664 \\
\hline 2 & 20320 & 25920 & 99520 & 881280 & 7520 & 2 & 1624 & 1696 \\
\hline 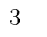 & 1920 & 27200 & 68480 & 3760 & 2080 & 688 & 224 & 1280 \\
\hline 4 & & & & & & & & 7072 \\
\hline 5 & 890240000 & -1693649 & 548946452480 & 1680 & 9011840 & 53248 & 2656 & 24668416 \\
\hline e & -48823359048253440 & 196736261750784000 & -294878887018168320 & & 673002645913600 & 182208 & 1376 & 2385672663859200 \\
\hline 7 & 49936 & -1727 & & & & & & -28893 \\
\hline 8 & -37157 & 00160 & 2400 & 13898 & 50400 & 3280 & 3952 & 18297 \\
\hline 9 & 12188408516444160 & -24921703427604480 & 30766294924001280 & -30666221514915840 & 21332885797601280 & -10156385601847296 & 2900703018221568 & -460660386299904 \\
\hline
\end{tabular}

Table 2.: Series for the logarithm of the partition function $\ln Z$ per dimer

$$
\ln Z(\lambda ; \beta)=\ln Z_{0}+\sum_{n=1}(-\lambda)^{n} \frac{1}{12^{n} n !} f_{n}(\beta)
$$

$$
f_{n}(\beta)=\sum_{k=0}^{n} \sum_{l=0}^{n+1} c_{k l} \beta^{k} Z_{0}^{-l} .
$$

Here $Z_{0}=1+3 \exp (-\beta)$ is the partition function of an isolated dimer.

$f_{2}$ :

\begin{tabular}{l|r|r}
$1 \backslash \mathrm{k}$ & 1 & 2 \\
\hline 0 & -24 & 96 \\
1 & 48 & -48 \\
2 & 192 & -48
\end{tabular}

$f_{3}:$

\begin{tabular}{l|r|r|r}
$\mathrm{l} \backslash \mathrm{k}$ & 1 & 2 & 3 \\
\hline 0 & 432 & 864 & -288 \\
1 & -864 & -1728 & 576 \\
2 & -3456 & 864 & -288
\end{tabular}

\begin{tabular}{l|r|r|r|r}
$\mathrm{l} \backslash \mathrm{k}$ & 1 & 2 & 3 & 4 \\
\hline 0 & -41760 & -55296 & -13824 & -2304 \\
1 & 158400 & 160704 & -24192 & -77760 \\
2 & 138240 & -430272 & 10368 & 205632 \\
3 & -506880 & 988416 & -110592 & -122112 \\
4 & 368640 & -663552 & 138240 & -3456
\end{tabular}

$f_{5}:$

\begin{tabular}{l|r|r|r|r|r}
$\mathrm{Mk}$ & 1 & 2 & 3 & 4 & 5 \\
\hline 0 & 2859840 & 4199040 & 2782080 & 103680 & 109440 \\
1 & -12476160 & -17210880 & -10091520 & 1105920 & 668160 \\
2 & -6324480 & 38413440 & 10938240 & 2799360 & -2609280 \\
3 & 47969280 & -56920320 & -864000 & -9331200 & 2776320 \\
4 & -24330240 & 31518720 & -2764800 & 5322240 & -944640
\end{tabular}




\begin{tabular}{l|r|r|r|r|r|r}
$1 \backslash \mathrm{k}$ & 1 & 2 & 3 & 4 & 5 & 6 \\
\hline 0 & -569462400 & -672675840 & -310832640 & -63452160 & 6220800 & -6220800 \\
1 & 2689459200 & 2966492160 & 1260126720 & 296524800 & 70917120 & 183831552 \\
2 & -156245760 & -8726745600 & -187453440 & 7464960 & -673505280 & -444552192 \\
3 & -7881960960 & 16105236480 & -5395921920 & -610053120 & 2198845440 & -16312320 \\
4 & 9728501760 & -23978488320 & 15582689280 & -1792005120 & -2801848320 & 741325824 \\
5 & -9289728000 & 24020582400 & -19654410240 & 4663111680 & 1186099200 & -518676480 \\
6 & 4459069440 & -9714401280 & 8705802240 & -2501591040 & 13271040 & 60604416
\end{tabular}

$f_{7}:$

\begin{tabular}{l|r|r|r|r|r|r|r}
$1 \backslash \mathrm{k}$ & 1 & 2 & 3 & 4 & 5 & 7 & 7 \\
\hline 0 & 89047395360 & 110724485760 & 58990498560 & 16056714240 & -672053760 & -721405440 & 116024832 \\
1 & -440447414400 & -531234547200 & -287505469440 & -81453496320 & 3779758080 & 675827712 & -6399074304 \\
2 & 117483307200 & 1579679781120 & 317464842240 & 85326151680 & 814302720 & -17347696128 & 21352536576 \\
3 & 1279789580160 & -2813668462080 & 422485217280 & -152130908160 & 20591262720 & 31405667328 & -21181160448 \\
4 & -1955637250560 & 3737580900480 & -1687259185920 & 495232496640 & -146069360640 & 28659972096 & -1691698176 \\
5 & 1533362503680 & -3133193011200 & 1945478062080 & -555514122240 & 209889792000 & -81196867584 & 12815953920 \\
6 & -545492828160 & 1050110853120 & -769653964800 & 192483164160 & -88333701120 & 38524502016 & -5012582400
\end{tabular}

$f_{8}:$

\begin{tabular}{l|r|r|r|r|r|r|r|r}
$l \backslash \mathrm{k}$ & 1 & 2 & 3 & & 5 & \\
\hline 0 & -28664815818240 & -32591048232960 & -14499570032640 & -3021936906240 & -239733043200 & 177006477312 & 26531463168 & 12850679808 \\
1 & 144608849925120 & 155543330073600 & 68811924142080 & 17838829532160 & 1926486374400 & -2269960132608 & -514036518912 & -249380305920 \\
2 & -60794389555200 & -518632755379200 & -59806624389120 & -30553999580160 & -13546484582400 & 10249704105984 & 3478877982720 & -918892071936 \\
3 & -366285335592960 & 1025042394378240 & -223731649290240 & 83800325468160 & 46315773665280 & -42114884345856 & -7230439931904 & 8011031556096 \\
4 & 639727016140800 & -1416307344076800 & 828567910195200 & -312900967249920 & -75109982330880 & 126097177006080 & -4625486659584 & -15544549767168 \\
5 & -579394111242240 & 1661222905896960 & -1523031536517120 & 748914182553600 & 7577120194560 & -200735322439680 & 34784356368384 & 10164135444480 \\
6 & 517910510960640 & -1681713886003200 & 1851925113077760 & -1122885281710080 & 166439411712000 & 149237092859904 & -444402769395712 & 819762315264 \\
7 & -442482378670080 & 1196838019399680 & -1343036042772480 & 912495052062720 & -217829258035200 & -36727534190592 & 21548808142848 & -2844450422784 \\
8 & 190443883069440 & -389401616056320 & 414800475586560 & -293686204170240 & 84466666045440 & -3913279340544 & -3065841451008 & 549492572160
\end{tabular}

